\title{
Adherence and awareness of the therapeutic intent of oral anticancer agents in an outpatient setting
}

\author{
MICHIO KIMURA ${ }^{1}$, KEIJI NAKASHIMA ${ }^{1}$, EISEKI USAMI ${ }^{1}$, MINA IWAI $^{1}$, TOSHIYA NAKAO $^{1}$, \\ TOMOAKI YOSHIMURA ${ }^{1}$, HIROMI MORI ${ }^{1}$ and HITOMI TERAMACHI ${ }^{2}$ \\ ${ }^{1}$ Department of Pharmacy, Ogaki Municipal Hospital, Ogaki, Gifu 503-8502; \\ ${ }^{2}$ Laboratory of Clinical Pharmacy, Gifu Pharmaceutical University, \\ Ogaki, Gifu 501-1196, Japan
}

Received May 13, 2014; Accepted February 17, 2015

DOI: $10.3892 / 01.2015 .3027$

\begin{abstract}
The aim of the present study was to clarify the adherence and awareness of oral anticancer agents by type and therapeutic purpose in outpatients prescribed with tegafur/gimeracil/oteracil potassium (S-1) or capecitabine. Outpatients undergoing treatment with the S-1 or capecitabine oral anticancer agents at Ogaki Municipal Hospital (Ogaki, Japan) in June 2013 completed a questionnaire survey and the survey findings were evaluated. No significant differences in medication adherence were identified between the patients administered $\mathrm{S}-1$ and the patients administered capecitabine $(\mathrm{P}=0.4586)$. In addition, no significant differences were identified in therapeutic purpose between adjuvant therapy, and advanced and recurrent therapies. However, for S-1 and capecitabine, medication adherence was significantly higher in those undergoing combination therapy compared with those undergoing monotherapy $(\mathrm{P}=0.0046)$. In addition, for patients taking $\mathrm{S}-1$, the median age for good adherence was significantly lower than that for insufficient adherence $(66.1 \pm 10.5$ vs. $72.1 \pm 7.9$ years, respectively; $\mathrm{P}=0.0035$ ). Furthermore, a significant negative correlation was identified between the awareness score of research regarding the medication and age $(\mathrm{n}=109 ; \mathrm{P}=0.0045)$. In conclusion, for patients treated with $\mathrm{S}-1$ or capecitabine, the type and therapeutic purpose of oral anticancer agents did not affect medication adherence. Elderly patients expressed a low interest in medications and typically exhibited insufficient medication adherence. Therefore, patient guidance by pharmacists is important, as it may result in improved medication adherence and an improved understanding of the treatment side-effects in patients self-administering prescribed drugs.
\end{abstract}

Correspondence to: Mr. Michio Kimura, Department of Pharmacy, Ogaki Municipal Hospital, 4-86 Minaminokawa-cho, Ogaki, Gifu 503-8502, Japan

E-mail: kimkim0305nao@yahoo.co.jp

Key words: adherence, patient awarencess, oral anticancer agent, adjuvant, progressive

\section{Introduction}

It is important that patients understand the effects of prescribed medicines, as well as the side-effects and methods of administration, as good medication adherence can lead to more effective treatment, improved safety and continuity of treatment in cancer chemotherapy (1-3). Therefore, it is necessary to investigate medication adherence in patients undergoing chemotherapy with consideration of their understanding of the side-effects.

Multiple studies regarding the adherence of patients to oral anticancer agents have been reported $(1,2,4-8)$. However, thus far, regimen adherence to tegafur/gimeracil/oteracil potassium (S-1) or capecitabine and multiple tyrosine kinase inhibitors have not been examined. Furthermore, the level of awareness of these medicines, which often corresponds to non-adherence by patients, has yet to be investigated. The side-effects of the aforementioned oral anticancer agents are characterized by the development of skin disorders and digestive system side-effects (9-11). In our previous study, a questionnaire survey was conducted to evaluate factors that may affect medication adherence in patients taking oral anticancer agents (3). It was identified that the medication compliance of patients self-administering oral anticancer agents was adequate, however, medication adherence was insufficient. These findings indicate that a patients' interest in their own treatment should be encouraged and interventions should occur according to the condition of the individual patient. However, to the best of our knowledge, no studies have thus far been conducted regarding medication adherence and awareness with regard to the type and therapeutic purpose of individual oral anticancer agents. In particular, as adjuvant chemotherapy is used for the prevention of post-operative recurrence, we propose that medication adherence and awareness is reduced in patients undergoing adjuvant chemotherapy in comparison with those undergoing advanced and recurrent chemotherapeutic regimens.

Therefore, the aim of the present study was to investigate the adherence to medication, and the awareness of oral anticancer agents with regard to the type and therapeutic purpose in outpatients who were self-administering S-1 or capecitabine. 
Table I. Questionnaire analysis items.

Topic

A. Medication adherence

B. Dosing awareness

C. Medication awareness

D. Understanding of the stage of the disease

E. Sense of trust

F. Expectations and attitude
1, I understand how to take the medication

2, I know the effect (efficacy) of the medication

3 , I know the side effects of the medication

4, I understand the current therapy

5, I agree with the current treatment policy

6 , I have forgotten to take the medication or I have mistakenly taken the medication

7 , I take care not to forget my medication

8 , I believe the medication is necessary for me

9, I believe the medication is effective

10 , I have actively researched about my medication

11 , I worry about side-effects

12 , I would like to talk to someone further about the medication

13 , I have a good understanding of my disease

14 , I trust the attending physician

15 , I trust the pharmacist

16 , I hope that the medication is valuable in curing the disease or that it will be able to improve my quality of life

17, I have a positive attitude towards the disease

\section{Patients and methods}

Survey topics and analysis method. A questionnaire survey was administered to 172 outpatients undergoing treatment with oral anticancer agents at Ogaki Municipal Hospital (Ogaki, Japan) in June 2013. The present study examines the survey findings for outpatients taking S-1 or capecitabine. The assessed questionnaire items included: Medication adherence (six items total), with one item each for the dosing method, effect of medication, side-effects, understanding of the treatment method, treatment policy and compliance; dosing awareness, (one item); medication awareness (five items); understanding of the stage of the disease (one item); sense of trust (two items); and expectations and attitude (two items) (Table I). The present study was approved by the Institutional Review Board of Ogaki Municipal Hospital. The study details were explained to the patient cohort and informed consent was obtained. Furthermore, personal information was protected in the aggregated data.

Medication adherence. The survey items were rated on a five-point Likert scale, with 5 being 'Yes', 4 being 'I think so', 3 being 'I cannot say either way', 2 being 'I don't think so', and 1 being 'No'. Participants were divided into two groups: i) The good medication adherence group, consisting of patients that adhered to their medication regimens (a score of $>4$ on all items); and ii) the insufficient medication adherence group, consisting of patients that adhered to their medication regimens (a score of $\leq 3$ on all items). Each measure of medication adherence was compared to the patient attributes.

Medication awareness. To examine factors that may affect the medication awareness score, the correlation analysis was performed between each topic score, and age and dosing timespan. In addition, therapeutic purpose (adjuvant therapy or advanced and recurrent therapies) was compared for each questionnaire topic.

Statistical analysis. The Mann-Whitney U test was performed to compare the two groups. In all significance tests, $\mathrm{P}<0.05$ was used to indicate a statistically significant difference. $\chi^{2}$ tests were conducted to examine differences in medication adherence with regard to patient attributes. In addition, Spearman's rank correlation coefficient was used to explore the correlations between medication awareness scores, and age and dosing timespan. All statistical analyses were performed using JMP 8 software (SAS Institute Inc., Cary, NC, USA).

\section{Results}

Patient background. Patient characteristics are indicated in Table II. The chemotherapeutic agents, and the numbers of patients self-administering S-1 or a combination of S-1 and other agents were as follows: $S-1, n=69 ; S-1$ plus cisplatin, $\mathrm{n}=11 ; \mathrm{S}-1$ plus irinotecan, $\mathrm{n}=2$; and $\mathrm{S}-1$ plus gemcitabine, $\mathrm{n}=1$. Furthermore, the numbers of patients self-administering capecitabine were as follows: Capecitabine, $n=12$; capecitabine plus oxaliplatin (XELOX), $n=7$; XELOX plus bevacizumab, $n=5$; capecitabine plus lapatinib, $n=1$; and capecitabine plus cisplatin, $n=1$. The number of participants undergoing monotherapy was significantly higher among those taking S-1 compared with those taking capecitabine (69 vs. 12 participants; $\mathrm{P}=0.0012$ ). However, no significant difference was observed between S-1 and capecitabine in association with the other investigated patient characteristics. 
Table II. Patient characteristics.

\begin{tabular}{|c|c|c|}
\hline Characteristic & S-1 (n=83) & Capecitabine $(n=26)$ \\
\hline \multicolumn{3}{|l|}{ Age, years } \\
\hline Mean \pm SD & $67.2 \pm 9.9$ & $63.5 \pm 12.2$ \\
\hline \multicolumn{3}{|l|}{ Gender, $\mathrm{n}$} \\
\hline Male/female & $48 / 35$ & $13 / 13$ \\
\hline \multicolumn{3}{|l|}{ Cancer type, $n$} \\
\hline Gastric & 44 & 2 \\
\hline Colorectal & 8 & 17 \\
\hline Liver & 5 & 0 \\
\hline Pancreatic & 9 & 0 \\
\hline Esophageal & 8 & 0 \\
\hline Breast & 2 & 7 \\
\hline Others & 7 & 0 \\
\hline \multicolumn{3}{|l|}{ Stage, $\mathrm{n}$} \\
\hline Adjuvant & 28 & 10 \\
\hline Progressive & 55 & 16 \\
\hline \multicolumn{3}{|l|}{ Chemotherapy regimen, $n$} \\
\hline S-1 only & $69(\operatorname{adj}, 28 ; \operatorname{prg}, 41)^{\mathrm{a}}$ & - \\
\hline S-1 plus cisplatin & 11 (adj, 0; prg: 11) & - \\
\hline S-1 plus oxaliplatin & $2(\operatorname{adj}, 0 ; \operatorname{prg}, 2)$ & - \\
\hline S-1 plus gemcitabine & $1(\operatorname{adj}, 0 ; \operatorname{prg}, 1)$ & - \\
\hline Capecitabin & - & $12(\operatorname{adj}, 3 ; \operatorname{prg}, 9)$ \\
\hline XELOX & - & $7(\operatorname{adj}, 6 ; \operatorname{prg}, 1)$ \\
\hline XELOX plus BV & - & $5(\operatorname{adj}, 1 ; \operatorname{prg}, 4)$ \\
\hline Capecitabin plus lapatinib & - & $1(\operatorname{adj}, 0 ; \operatorname{prg}, 1)$ \\
\hline Capecitabin plus cisplatin & - & $1(\operatorname{adj}, 0 ; \operatorname{prg}, 1)$ \\
\hline \multicolumn{3}{|l|}{ Dosing timespan, months ${ }^{\mathrm{b}}$} \\
\hline Median (range) & 144 (24-1989) & $133(54-641)$ \\
\hline
\end{tabular}

${ }^{a} \mathrm{~S}-1$ has been used in monotherapy rather than capecitabine $(\mathrm{P}=0.0012)$. For other items, no difference was observed between $\mathrm{S}-1$ and capecitabine. ${ }^{b}$ Dosing timespan indicates the period of time up to the date the survey was conducted. SD, standard deviation; S-1, tegafur/gimeracil/oteracil potassium; adj, adjuvant; prg, progressive; XELOX, capecitabin plus oxaliplatin; BV, bevacizumab.

Medication adherence with regard to patient attributes in patients self-administering $S-1$ or capecitabine. The medication adherence scores with regard to the patient attributes in patients self-administering S-1 or capecitabine are indicated in Table III. For patients taking S-1, good and insufficient adherence to medication occurred in 54 and 29 cases, respectively. In patients taking capecitabine, the counts were 16 and 10 cases, respectively. For medication adherence, no significant differences were identified between the patients taking S-1 and the patients taking capecitabine $(\mathrm{P}=0.4586)$. In addition, no significant differences were identified with regard to the therapeutic purpose between adjuvant therapy, and advanced and recurrent therapies.

For patients taking S-1, the data identified that the median age for good adherence was $66.1 \pm 10.5$ years. This was significantly lower than the median age identified for insufficient adherence $(72.1 \pm 7.9$ years; $P=0.0035)$. By contrast, no significant differences were identified in the median age for good adherence in patients taking capecitabine $(\mathrm{P}=0.3478)$.
Medication adherence in monotherapy versus combined therapy with regard to medication type. The comparison between medication adherence in patients undergoing monotherapy or combined therapy with $\mathrm{S}-1$ and capecitabine is indicated in Table IV. For the two therapeutic strategies, medication adherence was significantly higher in those undergoing combination therapy compared with those undergoing monotherapy $(\mathrm{P}=0.0046)$.

Medication awareness scores by therapeutic purpose. The awareness scores regarding medication in adjuvant chemotherapy or advanced and recurrent therapies are indicated in Table V. For topic number 10 ('research about medication'), no significant differences in awareness scores were identified between the patients undergoing S-1 adjuvant chemotherapy, and patients self-administering $\mathrm{S}-1$ advanced and recurrent therapies. However, for the medication awareness scores in patients taking capecitabine, the values were significantly higher in the adjuvant chemotherapy group (mean score, 4; 
Table III. Medication adherence and patient attributes in individuals taking S-1 or capecitabine ${ }^{\mathrm{a}}$.

\begin{tabular}{|c|c|c|c|c|c|c|}
\hline \multirow[b]{2}{*}{ Attribute } & \multicolumn{3}{|c|}{ S-1 } & \multicolumn{3}{|c|}{ Capecitabine } \\
\hline & $\begin{array}{c}\text { Good } \\
\text { adherence }(n=54)\end{array}$ & $\begin{array}{c}\text { Insufficient } \\
\text { adherence }(n=29)\end{array}$ & P-value & $\begin{array}{c}\text { Good } \\
\text { adherence }(n=16)\end{array}$ & $\begin{array}{c}\text { Insufficient } \\
\text { adherence }(n=10)\end{array}$ & P-value \\
\hline \multicolumn{7}{|l|}{ Age, years } \\
\hline Mean \pm SD & $66.1 \pm 10.5$ & $72.1 \pm 7.9$ & 0.0035 & $62.1 \pm 12.9$ & $66.3 \pm 11.7$ & 0.3478 \\
\hline \multicolumn{7}{|l|}{ Gender, $\mathrm{n}$} \\
\hline Male/female & $30 / 24$ & $18 / 11$ & 0.3769 & $8 / 8$ & $4 / 6$ & 0.5666 \\
\hline \multicolumn{7}{|l|}{ Stage, $\mathrm{n}$} \\
\hline Adjuvant/progressive & $15 / 39$ & $13 / 16$ & 0.2022 & $7 / 9$ & $3 / 7$ & 0.3905 \\
\hline \multicolumn{7}{|l|}{ Therapeutic agents, $\mathrm{n}^{\mathrm{b}}$} \\
\hline Mean $\pm \mathrm{SD}$ & $4.1 \pm 2.5$ & $4.9 \pm 2.8$ & 0.1973 & $3.1 \pm 1.7$ & $4.4 \pm 2.4$ & 0.1625 \\
\hline \multicolumn{7}{|l|}{ Dosing timespan, days } \\
\hline Median (range) & $132(26-1989)$ & $150(24-1292)$ & 0.7562 & $118(38-456)$ & $158(24-641)$ & 0.3748 \\
\hline
\end{tabular}

${ }^{a}$ No significant difference in adherence between $\mathrm{S}-1$ and capecitabine patients $(\mathrm{P}=0.4586)$. ${ }^{\mathrm{b}}$ Includes medication other than anticancer agents S-1, tegafur/gimeracil/oteracil potassium; SD, standard deviation.

Table IV. Medication adherence with regard to monotherapy or combined therapy with S-1 and capecitabine.

\begin{tabular}{|c|c|c|c|}
\hline \multirow[b]{2}{*}{ Therapeutic strategy } & \multicolumn{2}{|c|}{ Medication adherence } & \multirow[b]{2}{*}{ P-value } \\
\hline & Good, n & Insufficient, $\mathrm{n}$ & \\
\hline \multicolumn{4}{|l|}{ S-1 } \\
\hline Monotherapy & 38 & 27 & 0.0134 \\
\hline Combined therapy & 16 & 2 & \\
\hline \multicolumn{4}{|l|}{ Capecitabine } \\
\hline Monotherapy & 6 & 7 & 0.1131 \\
\hline Combined therapy & 10 & 3 & \\
\hline \multicolumn{4}{|l|}{ Total } \\
\hline Monotherapy & 44 & 34 & 0.0046 \\
\hline Combined therapy & 26 & 5 & \\
\hline
\end{tabular}

S-1, tegafur/gimeracil/oteracil potassium.

range, 1-5) compared with the advanced and recurrent therapies group (mean score, 1 ; range, $1-4)(\mathrm{P}=0.0253)$.

For topic number 11 ('worry about side-effects'), patients taking S-1 adjuvant chemotherapy scored significantly higher (mean score, 4; range, 1-5) compared with those taking $\mathrm{S}-1$ advanced and recurrent therapies (mean score, 3; range, 1-5) $(\mathrm{P}=0.0403)$. However, for patients taking capecitabine, no significant differences in awareness scores were identified with regard to therapeutic purpose.

Association between medication awareness scores, and age and dosing timespan. The association between medication awareness scores, age and dosing timespan were analyzed. For topic number 10 ('research about medication'), a significant negative correlation was identified between the awareness score and age $(n=109 ; r=-0.2735 ; P=0.0045)$. In addition, a significant negative correlation was identified between the score for topic number 11 ('worry about side-effects'), and age $(n=109 ; r=-0.2049 ; P=0.0339)$ and dosing timespan $(n=109$; $\mathrm{r}=-0.2701 ; \mathrm{P}=0.0050)$. No differences were observed among the other investigated topics.

\section{Discussion}

Cancer chemotherapy can be administered as oral single agents, injected single agents, or a combination of oral and injected agents (12-14). Cancer treatment with oral anticancer agents has the advantage of ease of delivery, however, as medication management is the responsibility of the family or patient, medication adherence can be a problem. In the present study, no significant differences were identified in the adherence to oral anticancer agents with regard to type (S-1 versus 
Table V. Score value of medication awareness in adjuvant theraphy or advanced and recurrent therapies.

\begin{tabular}{|c|c|c|c|c|c|c|}
\hline \multirow[b]{2}{*}{ Topic } & \multicolumn{2}{|c|}{$\mathrm{S}-1$, mean (range) } & \multirow[b]{2}{*}{ P-value } & \multicolumn{2}{|c|}{ Capecitabine, mean (range) } & \multirow[b]{2}{*}{ P-value } \\
\hline & $\begin{array}{l}\text { Adjuvant } \\
(\mathrm{n}=28)\end{array}$ & $\begin{array}{l}\text { Progressive } \\
\quad(n=55)\end{array}$ & & $\begin{array}{c}\text { Adjuvant } \\
(\mathrm{n}=10)\end{array}$ & $\begin{array}{l}\text { Progressive } \\
\quad(n=16)\end{array}$ & \\
\hline 1, Understanding of dosing method & $5(4-5)$ & $5(3-5)$ & 0.6528 & $5(4-5)$ & $5(3-5)$ & 0.4612 \\
\hline 2 , Understanding of effect & $5(1-5)$ & $5(1-5)$ & 0.7539 & $5(3-5)$ & $5(1-5)$ & 0.4473 \\
\hline 3 , Understanding of side effects & $5(1-5)$ & $5(1-5)$ & 0.4981 & $5(3-5)$ & $5(3-5)$ & 0.2830 \\
\hline 4 , Understanding of treatment method & $5(4-5)$ & $5(3-5)$ & 0.6107 & $5(3-5)$ & $5(3-5)$ & 0.8334 \\
\hline 5, Agreement of treatment policy & $5(3-5)$ & $5(3-5)$ & 0.7161 & $5(3-5)$ & $5(1-5)$ & 0.7641 \\
\hline 6, Compliance & $5(2-5)$ & $5(2-5)$ & 0.9612 & $5(4-5)$ & $5(1-5)$ & 0.2732 \\
\hline 7, Attention for forgetting medication & $5(4-5)$ & $5(3-5)$ & 0.3522 & $5(4-5)$ & $5(4-5)$ & 0.7031 \\
\hline 8, The medication is necessary & $5(1-5)$ & $5(3-5)$ & 0.7644 & $5(3-5)$ & $5(4-5)$ & 0.8612 \\
\hline 9, The medication is effective & $5(3-5)$ & $5(2-5)$ & 0.7358 & $5(3-5)$ & $5(3-5)$ & 0.2638 \\
\hline 10, Research about medication & $1(1-5)$ & $1(1-5)$ & 0.8965 & $4(1-5)$ & $1(1-4)$ & 0.0253 \\
\hline 11 , Worry about side effect & $4(1-5)$ & $3(1-5)$ & 0.0403 & $4(1-5)$ & $4(2-5)$ & 0.6066 \\
\hline 12 , Desire to consult about medication & $2(1-5)$ & $1(1-5)$ & 0.9862 & $3(1-5)$ & $3(1-5)$ & 0.5665 \\
\hline 13, Understanding of disease & $5(3-5)$ & $5(2-5)$ & 0.9661 & $5(1-5)$ & $5(4-5)$ & 0.6756 \\
\hline 14 , Confidence in doctor & $5(4-5)$ & $5(1-5)$ & 0.0986 & $5(4-5)$ & $5(3-5)$ & 0.6749 \\
\hline 15 , Confidence in pharmacist & $5(2-5)$ & $5(1-5)$ & 0.6200 & $5(3-5)$ & $5(3-5)$ & 0.1065 \\
\hline 16, Hope of medication & $5(2-5)$ & $5(2-5)$ & 0.3482 & $5(4-5)$ & $5(2-5)$ & 0.3033 \\
\hline 17 , Positive attitude & $5(3-5)$ & $5(3-5)$ & 0.1222 & $5(4-5)$ & $5(1-5)$ & 0.1279 \\
\hline
\end{tabular}

S-1, tegafur/gimeracil/oteracil potassium.

capecitabine) or therapeutic purpose (adjuvant therapy versus advanced and recurrent therapies). In addition, with regard to the association between medication adherence and age, medication adherence was considered to be insufficient in elderly patients taking S-1. However, no significant adherence differences were identified with regard to age in patients taking capecitabine (Table III). Furthermore, Hasegawa et al (15) and Tsuboi et al (16) reported greater medication adherence in elderly patients compared with young patients. Therefore, it should be considered that medication adherence may be largely dependent on factors other than age.

The dosing timespans for patients taking S-1 or capecitabine did not appear to affect medication adherence in the present study. In a previous study (3), patients with longer courses of treatment (dosing timespan, 220 days) demonstrated insufficient medication adherence. By contrast, the dosing timespan for the S-1 or capecitabine survey participants of the present study was markedly shorter, at 150 days (3). Tsang et al (17) reported that adherence decreases rapidly $\sim 6$ months after the commencement of treatment. Therefore, the comparatively short dosing timespan in the present study exhibited no effect on medication adherence.

With regard to medication adherence for S-1 and capecitabine monotherapy or combined injection treatment strategies, it was identified that medication adherence in patients taking S-1 was insufficient in monotherapy as opposed to combined therapy. Combined oral and injection chemotherapy was performed at the outpatient treatment center of Ogaki Municipal Hospital, and continual patient guidance by pharmacists and nurses is provided to such patients. By contrast, such guidance is not currently offered by pharmacists for patients undergoing an oral monotherapy regimen at Ogaki Municipal Hospital. Therefore, the present study proposes that patient guidance, or lack thereof, affected the medication adherence of patients taking oral anticancer agents.

For medication awareness in patients taking S-1 (Table V), concern regarding side-effects was more common in adjuvant chemotherapy patients compared with patients undergoing advanced and recurrent therapies. For patients taking capecitabine, such a difference was not apparent. A possible explanation for this result is that the dosing timespan of patients who took S-1 as an adjuvant chemotherapy was shorter compared with advanced and recurrent therapies (105 vs. 207 days), indicating that patients with short dosing times worried more about possible side-effects. Therefore, the present study concludes that worrying about side-effects is affected more by dosing timespan than by therapeutic purpose.

Patients taking capecitabine in adjuvant therapy scored higher on topic number 10 ('I have actively researched about my medication'; Table V), indicating that patients undergoing adjuvant therapy examined the medications they were taking more thoroughly compared with those undergoing other types of therapy. For patients receiving combined therapy with capecitabine, the number of patients in the adjuvant chemotherapy or advanced and recurrent chemotherapies groups were as follows: Adjuvant chemotherapy, 7/10 cases; advanced and recurrent chemotherapies, 6/16 cases. Therefore, as previously mentioned, continued patient guidance by pharmacists in outpatient treatment centers may lead patients to take a greater interest 
in their own medications. In patients receiving S-1 therapy, no guidance was received, as S-1 was predominantly used as a monotherapy. Accordingly, it was identified that S-1 patients did not conduct thorough research of their medication.

With regard to the association between awareness scores for topic number 11 ('worry about side-effects') and age, the correlation was weak; however, the tendency was for younger patients to worry about side-effects more and research the administered medications more thoroughly. By contrast, elderly patients displayed low interest in the prescribed medication and no anxiety regarding the possible side-effects. The low interest in medications by elderly patients may indicate that their ability to manage medication side-effects is lacking. In our previous study (3), patients with insufficient medication adherence did not fully understand the possible side-effects. In cancer chemotherapy, if the patient does not understand the side-effects and the methods to assuage them, then the safety of the patient and the continuity of the treatment strategy is threatened. Therefore, it is important to encourage a patient's interest in their own treatment medication. The results of the present study indicate that continual pharmacist guidance may improve patient management of possible therapeutic side-effects.

In conclusion, the present study identified that for the patients taking S-1 or capecitabine, the type and therapeutic purpose of the oral anticancer agents did not appear to significantly affect medication adherence. However, differences in medication adherence by therapy type (monotherapy versus combined therapy) were identified. In addition, elderly patients expressed low interest in their assigned medications and tended to exhibit insufficient medication adherence. Therefore, we propose that with continued medication guidance, elderly patients taking oral anticancer agents may be more interested in learning about the chemotherapeutic agent they are taking. Thus, patient guidance by pharmacists is important, as it may lead to improved medication adherence and understanding of the possible side-effects.

\section{References}

1. Wu EQ, Guerin A, Yu AP, Bollu VK, Guo A and Griffin JD Retrospective real-world comparison of medical visits, costs, and adherence between nilotinib and dasatinib in chronic myeloid leukaemia. Curr Med Res Opin 26: 2861-2869, 2010.

2. Reginster JY: Adherence and persistence: impact on outcomes and health care resources. Bone 38 (Suppl 2): S18-S21, 2006.

3. Kimura M, Usami E, Iwai M, Nakao T, Yoshimura T, Mori H, Sugiyama T and Taramachi H: Oral anticancer agent medication adherence by outpatients. Oncol Lett 8: 2318-2324, 2014.
4. Macintosh PW, Pond GR, Pond BJ, Leung V and Siu LL: A comparison of patient adherence and preference of packaging method for oral anticancer agents using conventional pill bottles versus daily pill boxes. Eur J Cancer Care (Engl) 16: 380-386, 2007.

5. Marin D, Bazeos A, Mahon FX, Eliasson L, Milojkovic D, Bua M, Apperley JF, Szydlo R, Desai R, Kozlowski K, et al: Adherence is the critical factor for achieving molecular responses in patients with chronic myeloid leukaemia who achieve complete cytogenetic response on imatinib. J Clin Oncol 28: 2381-2388, 2010.

6. Nilsson JL, Andersson K, Bergkvist A, Björkman I, Brismar A and Moen J: Refill adherence to repeat prescriptions of cancer drugs to ambulatory patients. Eur J Cancer Care (Engl) 15: 235-237, 2006.

7. Noens L, van Lierde MA, De Bock R, Verhoef G, Zachée P, Berneman Z, Martiat P, Mineur P, Van Eygen K, MacDonald K, et al: Prevalence, determinants, and outcomes of nonadherence to imatinib therapy in patients with chronic myeloid leukaemia: the ADAGIO study. Blood 113: 5401-5411, 2009.

8. Partridge AH, Archer L, Kornblith AB, Gralow J, Grenier D, Perez E, Wolff AC, Wang X, Kastrissios H, Berry D, et al: Adherence and persistence with oral adjuvant chemotherapy in older women with early-stage breast cancer in CALGB 49907: adherence companion study 60104. J Clin Oncol 28: 2418-2422, 2010.

9. Sakuramoto S, Sasako M, Yamaguchi T, et al; ACTS-GC Group: Adjuvant chemotherapy for gastric cancer with S-1, an oral fluoropyrimidine. N Engl J Med 357: 1810-1820, 2007.

10. Gore ME, Szczylik C, Porta C, et al: Safety and efficacy of sunitinib for metastatic renal-cell carcinoma: an expanded-access trial. Lancet Oncol 10: 757-763, 2009.

11. Escudier B, Eisen T, Stadler WM, et al: Sorafenib for treatment of renal cell carcinoma: Final efficacy and safety results of the phase III treatment approaches in renal cancer global evaluation trial. J Clin Oncol 27: 3312-3318, 2009.

12. Ruddy K, Mayer E and Partridge A: Patient adherence and persistence with oral anticancer treatment. CA Cancer J Clin 59: 56-66, 2009.

13. Russmann S, Curkovic I and Huber M: Adverse reactions and risks associated with non compliance. Ther Umsch 67: 303-307, 2010 (In German).

14. Winkeljohn D: Adherence to oral cancer therapies: nursing interventions. Clin J Oncol Nurs 14: 461-466, 2010.

15. Hasegawa K, Kuritani Y, Adachi A, Shinke K, Nishii S and Fujita Y: Improvement of drug compliance and pharmaceutical care - types of drug and dosing regimens desired by patients. Jpn J Pharm Health Care Sci 34: 800-804, 2008 (In Japanese).

16. Tsuboi K, Teramachi H, Kuzuya Y, Mizui T, Goto C and Tsuchiya T: Survey of the patients' consciousness affecting medication adherence. Jpn J Pharm Health Care Sci 38: 522-533, 2012. (In Japanese).

17. Tsang J, Rudychev I and Pescatore SL: Prescription compliance and persistency in chronic myelogenous leukaemia (CML) and gastrointestinal stromal tumor (GIST) patients (pts) on imatinib (IM). J Clin Oncol (Meeting Abstracts; Suppl) 24: 6119, 2006. 